\title{
La nouvelle organisation du notariat dans le Morbihan à l'époque révolutionnaire
}

\section{Martine Rouellé}

\section{(2) OpenEdition}

\section{Journals}

Édition électronique

URL : http://journals.openedition.org/abpo/914

DOI : 10.4000/abpo.914

ISBN : 978-2-7535-1501-7

ISSN : 2108-6443

Éditeur

Presses universitaires de Rennes

Édition imprimée

Date de publication : 30 mars 2006

Pagination : 111-121

ISBN : 978-2-7535-0273-4

ISSN : 0399-0826

\section{Référence électronique}

Martine Rouellé, "La nouvelle organisation du notariat dans le Morbihan à l'époque révolutionnaire », Annales de Bretagne et des Pays de l'Ouest [En ligne], 113-1 | 2006, mis en ligne le 30 mars 2008, consulté le 20 avril 2019. URL : http://journals.openedition.org/abpo/914 ; DOI : 10.4000/abpo.914 


\title{
La nouvelle organisation du notariat dans le Morbihan à l'époque révolutionnaire
}

\author{
Martine RouELLE \\ Doctorante en histoire \\ SOLITO - Université de Bretagne-Sud
}

La Révolution de 1789 ébranle les institutions de l'Ancien Régime. Tous les principes sur lesquels la monarchie a vécu pendant de longs siècles sont abolis le 4 août 1789. Ces événements provoquent de justes craintes chez les notaires quant à l'existence même de leur fonction. Après une période de latence qui ne fait qu'augmenter l'inquiétude, surtout celle des notaires seigneuriaux puisque le régime féodal n'existe plus, apparaît une réforme du notariat. "La réforme du notariat est venue tard, après celle de l'organisation judiciaire et celle de la profession d'avocat. Le maître d'œuvre n'est autre que le notaire Frochot, rapporteur d'un projet qui, tout en sacrifiant aux idées du temps, protège du mieux possible les intérêts de ses confrères notaires ${ }^{1}$. " Le rôle majeur du notaire n'est pas remis en cause, mais il faut opérer une réorganisation. " Le salmigondis notarial de l'Ancien Régime ne pouvait pas survivre très longtemps à la "Révolution des Juristes", à la réorganisation administrative et à l'abolition de la vénalité des offices ${ }^{2}$. " Le projet est adopté le 29 septembre et signé par le Roi le 6 octobre 1791 .

Cette loi comporte 96 articles. Les grands axes des cinq principaux articles seront évoqués, ainsi que les aménagements ultérieurs, accompagnés d'exemples in vivo pris dans la série $L$ des Archives départementales du Morbihan. Le contexte politique résonne de manière prégnante sur les modalités d'application de cette réforme et principalement en ce qui concerne les certificats de civisme, sésame indispensable à tout exercice.

1. SICARD, Germain, "Les notaires toulousains à l'épreuve de la Révolution " dans : Notaires, notariat et société sous l'Ancien Régime, dans LafFonT, Jean-Luc (dir.), actes du colloque de Toulouse, 15-16 décembre 1989, Toulouse, Presses universitaires du Mirail, 1990, p. 164.

2. BARRIERE, Jean-Paul, « Notables ou professionnels? 700 notaires de Haute-Garonne au XIXe siècle ", Thèse sous la direction de M. Augulhon, Paris I, 1993, 2385 p. dactyl. (voir p. 25). 


\section{Les suppressions}

Les premiers articles de ce décret sont consacrés à la création des notaires publics qui passe tout d'abord par la suppression des anciens notaires qu'ils soient royaux ou autres.

La vénalité et l'hérédité des offices royaux de notaires, tabellions, greffiers sont abolies. Les offices de notaires ou tabellions authentiques, seigneuriaux, apostoliques, sont supprimés. Ces divers officiers seront remplacés par des notaires publics. Il sera ensuite établi, dans tout le royaume, des fonctionnaires publics, chargés de recevoir tous les actes qui étaient précédemment du ressort des notaires supprimés et de leur donner le caractère d'authenticité attaché aux actes publics. Ces fonctionnaires porteront le nom de notaires publics ; ils seront institués à vie, et ils ne pourront être destitués que pour cause de prévarication préalablement jugée.

Plusieurs notaires seigneuriaux de la juridiction d'Hennebont avaient déjà réagi violemment à une annonce de la Feuille Hebdomadaire d'une ordonnance ${ }^{3}$ qui leur " porte l'atteinte la plus funeste ${ }^{4}$ " et sont déterminés à présenter une adresse à l'Assemblée Nationale tendant à son redressement. Outre les arguments juridiques qui leur font contester cette ordonnance, ils invoquent le fait que les notaires des juridictions seigneuriales font le lien avec les citoyens : les supprimer serait nuire à la Constitution ${ }^{5}$. Pour quelle raison, puisqu'ils sont maintenant tous égaux, sont-ils rayés de préférence aux notaires royaux? Le 4 mai, les notaires seigneuriaux de Pontivy se réunissent en assemblée pour joindre leur protestation à ceux d'Hennebont ${ }^{6}$.

Deux notaires, Avertain et Moraud, écrivent au procureur général syndic du département du Morbihan à Vannes le 23 mai 1791 pour expliquer leur infortune ${ }^{7}$. Le procureur souhaite attendre la parution de la loi pour

3. Arch. dép. du Morbihan, L 834, 2 avril 1791 : «[...] il a été ordonné que tous les notaires des ci-devant juridictions seigneuriales qui se trouvent supprimées, cesseraient toutes fonctions de Notariat jusqu'à la décision du Comité de Constitution, auquel il serait écrit, ainsi qu'à M. le Garde des Sceaux ".

4. Arch. dép. du Morbihan, L 834, Courrier accompagnant l'adresse des notaires seigneuriaux d'Hennebont le 20 juin 1791.

5. Jean Meyer montre que la puissance du notaire est multipliée dans le contexte breton par le retard de l'alphabétisation dans (MEYER, Jean, "La noblesse bretonne au XVIII siècle ", Paris, 1966, tome II, p. 630-640). Jean Meyer s'interroge sur le rôle du notaire comme " cheville de transmission entre la partie du groupe humain acculturé à la " galaxie Gutenberg " et celle de la population restée étroitement liée et incluse à l'intérieur d'une civilisation de l'oral " (MEYER, Jean, "La documentation notariale en Bretagne à l'époque moderne", dans Vogler, Bernard [dir.], Les Actes notariés : source de l'histoire sociale, XVI XIX siècles, actes du Colloque de Strasbourg, mars 1978, Strasbourg, ISTRA, 1979, 367 p. [voir p. 31]).

6. Arch. dép. du Morbihan, L 834.

7. L'article de Jeanne Grall, "Le notariat dans le Calvados pendant la Révolution ", Bulletin de la société des antiquaires de Normandie, tome 57, 1965, nous montre que des réactions similaires ont lieu dans ce département. 
leur répondre au sujet des nominations. Quelques lignes de ce courrier nous informent sur l'opinion portée sur la profession de notaire :

" [...] Il est digne de la révolution de rendre à la plus belle et à la plus importante des professions l'éclat et la dignité qui lui appartiennent, il est temps de la tirer de cet état d'abjection et d'avilissement où elle était tombée, en toute province, il faut qu'elle ne soit plus exercée que par des personnes qui à une probité intacte et à des mœurs pures joindront un esprit droit, un jugement sain, l'amour de l'étude et une connaissance approfondie des lois. Il serait à désirer qu'aucun notaire ne fut admis à en faire les fonctions qu'il ne fut homme de loi et soumis à une information de vie et mœurs ${ }^{8}$."

Nous retrouvons dans ces lignes les principaux griefs portés contre les notaires dans les cahiers de doléances. La demande des cahiers porte principalement sur la réduction des notaires royaux dans les campagnes, jugeant que beaucoup manquent de talent et d'honnêteté, réduisant certaines familles à la ruine. «Dans les cahiers de 1789 les doléances portaient essentiellement sur la réduction du nombre des charges, la limitation de la concurrence qu'elles se faisaient et la suppression des professions rivales $^{9}$. "Cependant, tous les cahiers ne soulèvent pas les problèmes liés au notariat. Line Skorka souligne que " ceux des bailliages de Tours et de Loches $[. .$.$] sont singulièrement muets " sur cette question { }^{10}$. Leur souhait est de voir arriver une réforme. Elle ferait des notaires, tant des campagnes que des villes, des hommes parfaitement instruits, dévoués à l'État, faisant honneur à leur profession et méritant la confiance du public.

L'article suivant précise que l'exercice des fonctions de notaire public sera incompatible avec celui des fonctions d'avoué et de greffier, et avec la recette des contributions publiques. C'est une remise en cause de bien des habitudes du notariat qui avaient coutume de cumuler les fonctions. Mais l'intérêt public risque aussi d'en subir les revers. Ainsi, les administrateurs du district de Pontivy mentionnent que cette incompatibilité va entraîner le choix vers de la fonction de notaire. Privé d'avoués, le cours de la justice risque d'être interrompu, car tous préféreront une activité indépendante et plus lucrative ${ }^{11}$. Un notaire de Ploërmel, en complet désarroi, déclare :

" [...] Je suis démonté, je vois pour l'avenir l'impossibilité d'élever convenablement mes enfants, peut être de les faire vivre, on perdrait la tête pour moins [...] Je n'ai, ni n'ai eu le désir que la conservation de mon état et voilà t-il pas qu'une révolution le culbute? Je la bénie et je la chérie cette révolu-

8. Arch. dép. du Morbihan, L 835.

9. Poisson, Jean-Paul, " Les notaires ", p. 60, dans Théodore ZeLDIN, Histoire des passions françaises 1848-1945, tome I : Ambition et amour, traduction de l'anglais par Paule Bolo et Denise Demoy, Paris, Seuil, 1980, 431 p.

10. «L'état du notariat tourangeau à la veille de la Révolution » dans : Notaires, notariat et société... op. cit., p. 151.

11. Arch. dép. du Morbihan, L 834, Lettre du 11 septembre 1793. 
tion parce qu'elle fait le bien général et qu'elle me paraît un chef-d'œuvre de raison et d'équité mais elle reverse toutes mes espérances et mes vues pour le bien-être de ma famille, n'importe je ne murmure pas et je me soumets ${ }^{12}$."

Malgré ses griefs, ce notaire avait tout intérêt à encenser la Révolution pour éviter que l'expression "perdre la tête " ne prenne une toute autre acceptation.

Choisir certes, mais comment le faire en toute tranquillité d'esprit? Comment prendre le bon parti dans cette période de grande agitation? Car « les notaires, à l'instar de leurs concitoyens, furent pris dans ce vaste mouvement " que fut la tourmente révolutionnaire ${ }^{13}$. Pierre Duportal, greffier à Ploërmel depuis 25 ans et notaire depuis 22 ans, déclare le 8 octobre 1792 que :

" [...] Quoiqu'il ignore les motifs d'incompatibilité décrété entre les fonctions de notaire et celle de greffier il déclare en se soumettant à la loi, qu'il entend dans ce moment continuer ses fonctions de greffier mais que comme il est journellement question de suppression des tribunaux de district et de nouveaux modes de réélection d'officiers, il réserve expressément à reprendre les fonctions de notaire publique ou même celle d'avoué conformément aux lois au cas que par événement il se trouve évincé de celle de greffier, il réserve aussi à faire liquider la finance de son dit office de notaire ${ }^{14}$."

Le nombre et le placement de ces fonctionnaires seront déterminés pour chaque département par le Corps législatif, d'après les instructions qui lui seront adressées par les directoires des départements.

Les critères pris en compte pour l'établissement des notaires publics seront, pour les villes, le chiffre de la population et, pour les campagnes, l'éloignement d'une ville, l'étendue du territoire et l'importance de la population. La mise en application de ces principes suscite de nombreuses réactions car "s'il est intéressant de placer des notaires à portée des citoyens qui ont besoin de faire rédiger leurs conventions, il n'est pas moins essentiel de n'en établir que dans les lieux pouvant être vraiment utiles ${ }^{15}$ ". Les courriers affluent de tous les districts. Les membres des tribunaux de districts établissent des listes par canton en justifiant le nombre de notaires à installer.

\section{L'établissement des notaires publics}

Les notaires seront, dans chaque département, répartis en trois classes. Les notaires de première classe seront admis de préférence à se faire rece-

12. Arch. dép. du Morbihan, L 834, Lettre du 24 septembre 1792 adressée à Gaillard procureur syndic.

13. JuLIEN, Jean-Paul, préface de Notaires, notariat et société..., op. cit.

14. Arch. dép. du Morbihan, L 834, Séance publique du 8 octobre 1792, Registre des délibérations du directoire du district de Ploërmel.

15. Arch. dép. du Morbihan, L 834, Circulaire Vannes le 22 octobre 1791 des administrateurs composant le Directoire du département du Morbihan. 
voir notaires publics dans les lieux où ils résident, mais ils ne pourront dans aucun cas opter pour une autre résidence. Quel que soit leur nombre, ils seront tous admis à exercer. Leur réduction ne s'opérera que par mort ou démission. Les notaires doivent faire savoir au procureur général syndic s'ils veulent être confirmés dans l'exercice de leurs fonctions en qualité de notaires publics. Passé le délai d'un mois, le défaut d'acceptation vaut renoncement à leur droit, et leur charge figure sur le tableau des places vacantes.

Cependant, le nombre de notaires fixé par la loi déborde les cadres de la réglementation. Le 28 brumaire an VI, le commissaire du directoire exécutif auprès du département du Morbihan informe le Ministre de la Justice que la loi du 13 août 1792 fixe le nombre de notaires pour le Morbihan à 127, alors qu'il en compte 207 sur le tableau général. Certes, on peut estimer que certains sont morts, mais le nombre reste de beaucoup supérieur à celui édicté par la loi. Ce qui suit est assez instructif quant au sentiment virulent que porte le commissaire sur la Bretagne :

" Je sais que cela provient des lois précédentes qui ont conservé presque tous les notaires cy-devant royaux et seigneuriaux, mais il est un moien sur de les rapprocher du nombre voulu par la loi, c'est d'appliquer l'arrêté du directoire exécutif du 14 vendémiaire dernier à tous les notaires de la république sans exception quelle que soit l'époque de leur nomination ou de leur provision; si les autres départements n'ont pas été plus prodigues de nomination que le notre, il y en a bien peu de créés depuis le régime constitutionnel [...] je crois vous assurer citoyen Ministre que la plus part des notaires sont presque partout encore très royaux et très seigneuriaux; on a trop longtemps fermé les yeux sur des hommes qui exercent dans la république une des fonctions les plus importantes et en même temps les plus influentes sur la grande masse du peuple qui dans presque toute la cy-devant Bretagne, est ignorante fanatique à l'excès et tout à fait illettrée; ce sont les notaires qui après les prêtres approchent de plus près cette portion si respectable de la nation ils ont avec elle les relations les plus intimes et qui la touchent le plus; ils sont presque partout et surtout dans les campagnes, les arbitres de ses intérêts les plus chers; il dépend d'eux d'y faire aimer et chérir les lois de la république; mais le langage qu'on entend chaque jour à cette portion ignorante et sans expérience ne prouve que trop qu'il est le fruit des leçons des notaires : les prêtres rebelles trouvent en eux des protecteurs; en reconnaissance des clientelles qu'ils leur procurent; je regarde qu'un des meilleurs moiens de republicaniser la masse des habitans des campagnes et même des villes et de leur faire chérir notre immortel gouvernement est de ne leur donner pour notaires que des républicains éprouvés et probes; qu'on épure donc sans délais cette espèce de corporation qui renferme dans son sein le poison de la royauté et de la féodalité qu'elle communique chaque jour à tout ce qui l'approche; cette reforme salutaire ne sera sûrement pas celle qui influera le moins sur l'esprit public, surtout dans les départements de l'ouest, j'abandonne ces réflexions rapides à votre sagesse et votre prudence ${ }^{16} \ldots$...

16. Arch. dép. du Morbihan, L 838. 
Les différents courriers de cette époque évoquent à de nombreuses reprises l'influence incontestable qu'exerce le notaire sur la population. Hormis les considérations d'ordre politique évoquées plus haut, on leur reconnaît un rôle éminent ${ }^{17}$ :

«Un des besoins les plus essentiels au public est un mediateur sage qui termine les contestations avec equité, qui previenne avec prudence celles qui pouraient naître par la suitte, enfin un espece d'arbitre ou de juge, qui par son exactitude à mettre les intentions des contractans dans tous leur jour, assure tout à la fois et la possession des biens et la tranquilité des familles ${ }^{18}$.

De manière positive encore, l'administration de Néant indique que la meilleure façon d'attacher les citoyens à la République est de les aider à contracter dans les meilleures conditions, donc de recourir à un notaire sans parcourir de longues distances. Car la répartition est déséquilibrée : certains soulignent qu'il existe beaucoup de fonctionnaires publics en certains endroits alors que d'autres en sont privés.

\section{Les minutes}

La nouvelle législation s'intéresse à l'instrument privilégié des notaires : les minutes.

"Celles des notaires supprimés seront mises en la garde des notaires publics établis dans la résidence la plus proche du lieu de leur dépôt actuel. Celles des notaires ayant cessé leur activité ou déplacé seront déposées chez celui des notaires publics qu'ils jugeront à propos de choisir parmi ceux établis dans le chef-lieu de résidence, et faire sur les recouvrements telles conventions que bon leur semblera ${ }^{19}$."

Le ministre de la Justice considère la conservation des minutes comme un objet important pour l'intérêt du public ${ }^{20}$. Souvent, les minutes restent dans les mains du démissionnaire ou de la famille du décédé. Les considérant comme une sorte de patrimoine, ils en disposent à leur gré, les vendant à tel ou tel notaire, quelquefois même à un individu sans titre. Les papiers de famille disparaissent, et le désordre qui peut en résulter est incalculable. Par contre, certains notaires, s'ils ne restent pas attachés à un office familial, s'efforcent de " conserver les minutes de leurs ancêtres. Comme de nombreux autres notaires poitevins, ils [les Bourbeau] préféraient, en effet, à la fiction immobilière de la charge, la matérialité des "notes et protocoles". Sources de profits du fait de la délivrance d'expédi-

17. Pierre Chaunu voit dans les notaires les " accoucheurs et gardiens de la mémoire des volontés accordées, les notaires sont un peu les prêtres et les médecins de la paix sociale " (CHAunu, Pierre, "Problèmes et méthodes d'analyse historique de l'activité notariale, $\mathrm{XV}^{\mathrm{e}}$-XIX ${ }^{\mathrm{e}}$ siècles ", dans Notaires, notariat et société..., op. cit., préface).

18. Arch. dép. du Morbihan, L 835, Arrêté en Municipalité à l'administration du canton de Bubry le deux nivôse l'an sixième de la République française.

19. Titre III de la législation de 1791.

20. Arch. dép. du Morbihan, L 838, sans date. 
tions, les minutiers des aïeux pouvaient fidéliser sur la longue durée une clientèle soucieuse de maintenir confidentialité de ses affaires dans une même famille ${ }^{21}$ ". Le ministre rappelle aux administrateurs du département d'exercer toute leur vigilance sur cette question. On évoque aussi le sort des minutes restées aux mains d'un notaire pris de démence comme Joubin de Caro ou Le Luc de Belle-Île ${ }^{22}$.

Le sort des anciens notaires étant réglementé, il s'agissait aussi de légiférer sur la nomination des nouveaux notaires publics. Les places des notaires publics ne pourront être occupées que par des sujets antérieurement désignés dans un concours public, qui aura lieu le premier septembre de chaque année, dans les villes chefs-lieux de département.

Lorsque le candidat se présente, les juges, au nombre de neuf, vérifient ses titres puis l'interrogent sur les lois constitutionnelles, les fonctions et devoirs de la profession. L'impétrant doit ensuite rédiger un acte notarié d'après les éléments donnés le jour du concours. Le jury délibère et ne déclare le notaire reçu que s'il obtient la majorité absolue des voix. Les élus voient leur nom figurer sur un tableau par ordre de mérite. À note identique, on donne la préférence à celui qui a fait le stage le plus long, et à stage égal, au plus âgé. Lorsqu'une étude devient vacante, le candidat nommé est celui qui est inscrit en premier sur le tableau. Cette méthode a le défaut de voir le dernier candidat d'une année nommé avant le premier de l'année suivante, ce qui ne favorise pas le mérite. "Les candidats même inscrits sur la liste, étaient tenus en attendant un emploi vacant de continuer leurs études et leur stage ${ }^{23}$. " Conscients de cet inconvénient, les juges font une deuxième sélection entre les inscrits, c'est celui qui a obtenu le plus de suffrage qui est choisi, quel que soit son rang antérieur. Le concours est finalement supprimé après que toutes les tentatives de le réformer aient échouées. De plus, il est détourné de son objectif premier puisque la nomination est soumise à l'obtention d'un certificat de civisme. Chaque candidat fait agir toutes les influences dont il pouvait disposer pour obtenir un meilleur classement.

La vénalité des offices supprimée, la loi doit indemniser les notaires de leurs charges. Les règles de remboursement, si elles favorisent les notaires de Paris, " n'assuraient pas aux notaires de province une indemnité suffisante $^{24}$ ».

21. JAHAN, Sébastien, "Notaires et notables poitevins : la dynastie Bourbeau XVIXIX ${ }^{\mathrm{e}}$ siècles ", Annales de Bretagne et des Pays de l'Ouest, 2001, Tome 108, n 1, p. 58.

22. Arch. dép. du Morbihan, L 838.

23. GASTON, Jean, "La communauté des notaires de Bordeaux (1520-1791)", Thèse de droit, Faculté de Droit de Bordeaux, 1913, réédition 1991, Toulouse, Presses universitaires du Mirail, p. 323.

24. MAGNAN, Jean-Louis, Le Notariat et la Révolution française, Imprimerie Forestié, 1952, $175 \mathrm{p}$. 


\section{Le remboursement des notaires royaux}

Tout titulaire d'office de judicature supprimé put se faire liquider individuellement, sans représenter l'état des dettes actives et passives de sa Compagnie, comme la Constituante l'avait tout d'abord prescrit.

Afin de ne pas trop grever le budget, les propriétaires d'offices supprimés ont la faculté d'employer la moitié du prix de leur finance en acquisition de domaines nationaux. Il est évident que les notaires ont plus intérêt à opter pour cette formule plutôt que d'accepter des assignats. La lecture des nombreux courriers dans lesquels les notaires se plaignent de leur forte difficulté à faire vivre correctement leur famille laisse supposer que l'acquisition des biens nationaux fut plus une mesure de survie qu'un acte politique. Ceci d'autant plus que le prix de base fut l'évaluation de $1771^{25}$, dans laquelle beaucoup avaient sous estimé le montant de leur office afin de diminuer l'impôt s'y rapportant. "Louis XV avait en effet ordonné l'estimation des offices dans l'édit de février 1771. Il avait précisé que la somme ainsi déclarée servirait de prix, voire d'indication, pour le remboursement en cas de suppression. Les officiers ne crurent pas à une telle éventualité et ils sous-évaluèrent leurs offices pour payer un droit casuel moins élevé. Vingt ans plus tard, ils allaient se retrouver victimes de leur fraude ${ }^{26}$. "

\section{Les certificats de civisme}

Les certificats de civisme sont prescrits dès le mois d'août 1792 pour les citoyens qui résident hors de leur domicile. Un décret de la Convention du $1^{\mathrm{er}}$ novembre $1792^{27}$ impose la fourniture d'un certificat de civisme aux notaires qui sont à nommer ainsi qu'à ceux qui sont maintenus provisoirement en fonction. Le 29 janvier 1793, il est décidé qu'aucun motif n'est à donner pour refuser les certificats de civisme aux notaires. Le 19 vendémiaire an III, les notaires destitués ou suspendus, faute de certificats de civisme, peuvent être réintégrés en les produisant. Le 11 thermidor an III, la Convention supprime la présentation des certificats de civisme pour échapper à la " loi des Suspects ". C'est donc un rétablissement de l'égal accès aux fonctions publiques garanti par la Déclaration des droits de 1789. Le climat politique se lit à travers cet énoncé, de l'extrême rigueur au libre exercice.

Le procureur général syndic Gaillard souhaite préciser la fonction des certificats de civisme et la manière dont ils doivent être rédigés. L'exécution de la loi qui astreint les notaires à fournir un certificat de civisme dans la huitaine de la rénovation des municipalités lui paraît :

25. Arch. dép. du Morbihan, B 1350, B 4682. Les notaires royaux de la sénéchaussée de Vannes les évaluent à mille livres, ceux de la sénéchaussée de Rhuys à 500 livres.

26. LAFON, Jacqueline Lucienne, La Révolution française face au système judiciaire d'Ancien Régime, préface de François Monnier, Genève/Paris, Droz, 2001, 464 p., voir p. 310.

27. Le Moniteur universel, $1792, \mathrm{n}^{\circ} 308$. 
" [d'une] importance majeure et devoir etre suivie avec la plus rigoureuse exactitude. Il faut enfin qu'on commence à dévoiler les mauvais citoyens et a leur rendre la justice qui leur est due, indignes de la confiance publique [...]. La substance des vrais amis de la révolution ne deviendra plus la proie des traîtres, le reigne des courtisans est fini, les magistrats d'un peuple libre ne connoissent que la vérité et ne savent point la taire [...]. Les certificats de civisme quilles vont donner aux notaires ne doivent pas être conçus en termes vagues par ce que pour être vérifiés par les corps administratifs il faut des objets susceptibles d'approfondissement".

Suit la liste des différents points qui doivent être examinés avant de délivrer le certificat : inscription sur les rôles des gardes nationales, dans les assemblées de communes, primaires ou électorales, jurés, s'ils n'ont pas de relation avec des prêtres réfractaires, bref s'ils sont de bons citoyens conformes aux principes de la Révolution ${ }^{28}$.

Le ministre de la Justice Lambrechts affirme ses positions quant à l'incivisme qui s'est manifesté dans plusieurs parties du corps politique : il faut installer de grandes mesures pour sauver la république. Après une " désinfection " des corps judiciaires et administratifs, le Directoire exécutif se doit d'étendre cette action aux corps secondaires : un état nominatif de la moralité et de la conduite politique des notaires doit être dressé.

" Il est parvenu au Directoire executif des révélations affligeantes sur ce point. Dans plusieurs départements où l'esprit public était vicié par un royalisme sans pudeur ou par un attiédissement presque aussi coupable, on a affecté de prêter aux places de notariat, des hommes qui se faisaient un mérite infame de se déclarer ennemis de la république, des hommes, qui, liés par une vieille intimité avec les déserteurs de la patrie, leur laissaient entrevoir, dans le mécanisme des transactions sociales, ces criminelles collusions, ces tournures perfides qui alimentent leurs espérances toujours coupables, toujours trompées et toujours renaissantes. Chargé par la Constitution (art. 195 et 196) d'éclairer et de rectifier les actes des autorités administratives, le Directoire executif ne doit pas laisser dans la machine sociale ce rouage ennemi, qui ne manquerait pas d'en retarder ou d'en altérer la marche. Il m'a fait un devoir de lui présenter les renseignements les plus prompts et les plus détaillés. J'aime à croire, Citoyens que votre Administration, en particulier, n'a point à se faire de reproches graves sur les diverses nominations qui ont eu lieu dans le notariat depuis deux ans; cependant si parmi les personnes qui ont été pourvues, il s'en trouvait malheureusement qui, par des principes répréhensibles ou même équivoques, appelassent la défiance publique, les bons citoyens qui sont parmi vous ne doivent pas hésiter à m'en instruire, en établissant, avec franchise et précision, des faits positifs ${ }^{29}$."

Le Corre, notaire à Plouay, nous éclaire sur le sentiment qui anime les hommes qui se font ainsi juger. Il montre la gravité qu'il y a à se prononcer irrévocablement sur le sort à venir d'un citoyen, sur son honneur, au risque de le dépouiller et de mettre en danger sa famille, " en un mot de mettre un

28. Arch. dép. du Morbihan, L 836, 1792.

29. Arch. dép. du Morbihan, L 838, an VI. 
homme la face contre terre ". Il préconise de prendre toute précaution et mesure et de se garder de la haine, des ambitions, de la jalousie et de la vengeance que l'on rencontre trop souvent dans les campagnes où les passions président et où l'opinion est influençable.

La délivrance de certificat de civisme rendue obligatoire pour les notaires le $1^{\mathrm{er}}$ novembre 1792 ne s'est pas faite de manière rigoureuse. $\mathrm{Si}$, au départ, il s'agit de faire le tri entre les patriotes et les autres, on découvre de nombreux témoignages qui parlent, de malveillance, de dépit. " Il suffisait d'une dénonciation, d'une animosité personnelle, pour qu'à un degré ou l'autre de la hiérarchie, la délivrance du certificat fût arrêtée ${ }^{30}$. " Quant aux sociétés populaires, leur rôle n'est pas négligeable puisqu'elles deviennent des officines de dénonciation et de vengeance.

Cette obligation désorganise profondément l'exercice de la profession. Alain Moreau rapporte que certains, par prudence, se dressent un acte par lequel ils se suspendent eux-mêmes de leur fonction, faute d'avoir obtenu le précieux document ${ }^{31}$. G. Maillard parle quant à lui du régime de l'arbitraire : "Quelqu'un voulait-il écarter un candidat des fonctions notariales, il lui était facile de faire agir ses influences auprès du Comité de ce dernier et de lui faire refuser son certificat ${ }^{32}$."

Devant l'imperfection et la non-application fréquente de la loi de 1791, nombreux sont les projets de réorganisation du notariat. En août 1792, l'Assemblée propose d'examiner à nouveau les dispositions de la loi de 1791. Le 5 messidor an IV, le conseil des Cinq-Cents nomme une commission pour étudier les modifications à apporter à la loi. Favard déclare le 23 germinal an VI au Conseil des Cinq-Cents « le notaire est encore ce qu'il était jadis, à la vénalité près; [ce qui n'était tout de même pas négligeable] il a conservé son activité, ses prérogatives et ses abus. Le notariat est debout au milieu des décombres de la Révolution ${ }^{33}$ ". Phrase paraissant plus une figure de style emphatique que le reflet de la réalité. Le 18 nivôse an VI, un message du Directoire insiste sur l'urgence d'une réorganisation du notariat. Le 23 germinal an VII un projet de résolution sur cette refonte vient devant les Cinq-Cents, mais il échoue lors de la séance du 28 prairial. Un nouveau projet est préparé dont le vote paraît assuré, lorsque survient le 18 brumaire. Usages et habitudes se perpétuent jusqu'à la loi du ventôse an XI. Le Consulat, par la loi du 25 ventôse an XI, donne au notariat sa nouvelle organisation, celle que les notaires connaissent encore aujourd'hui.

30. FoIRET, Faustin, Une Corporation parisienne sous la Révolution (les notaires), Paris, Champion, 1912, p 96

31. MoreaU, Alain, Les Métamorphoses du scribe, Histoire du Notariat français, Perpignan, Socapress-RCI, 1989, p. 163.

32. MAILlARD, G., Le Notariat sous la Révolution, Thèse de doctorat 24 novembre 1908 , Lille, Imprimerie Robbe, 175 p.

33. Citée par Louis FouRREY dans Les conditions d'accès au notariat, étude historique et critique, Thèse de doctorat du 1 ${ }^{\mathrm{er}}$ juin 1933, Paris, Domat-Monchrestien, 1933, p. 86. 


\section{RESUME}

L'anéantissement du système d'Ancien Régime par la Révolution française est marqué par la suppression de la vénalité des offices. La Constituante se donne pour mission de réformer toutes les institutions. L'organisation du notariat n'échappe donc pas à cette refonte. Le décret du 29 septembre-6 octobre 1791 pose les bases de ce nouvel ordonnancement par la création de notaires publics. Ils sont très vite soumis à l'obtention d'un certificat de civisme pour pouvoir exercer. Le climat révolutionnaire pèse de tout son poids sur cette période de changement politique et d'instabilité économique jusqu'à la promulgation de la loi organisant définitivement le notariat en ventôse an XI.

\section{ABSTRACT}

The shattering of the Ancien Régime by the French Revolution was marked by the abolition of the venality of offices. The Constituante took for mission the reformation of all the institutions. The organization of the notary profession was not exempt from this overhaul. The statutory order of September 29-October 9, 1791 laid the foundations of the new layout by the creation of notaries public. They were quickly subjected to obtaining an attestation of civic-mindedness to be able to practise. The revolutionnary climate weighed heavily on this period of political change and economic instability until the promulgation of the law which definitively organized the profession of notaries in ventôse an XI. 